\title{
SÉANCE DU 28 JANVIER 1916
}

\author{
Présidence de M. P.-A. Dangeard.
}

M. F. Moreau, vice-secrétaire, donne lecture du procèsverbal de la dernière séance, dont la rédaction est adoptée.

M. le Président a le regret d'annoncer à lả Société la mort de M. Heckel, correspondant de l'Institut, ancien professeur à la Falculté des Sciences de Marseille, et rappelle lés travaux scientifiques du défunt.

M. F. Moreau, en son nom et au nom de $M^{\text {me }}$ Moreau fait la communication suivante :

\section{La structure des Cyanophycées symbiotes des Peltigéracées;}

Par M. et Mme Fernand Moreau.

Les Champignons des Lichens de la famille des Peltigéracées vivent souvent en symbiose avec des Algues de la famille des Nostocacées. Celles-ci contractent avec le Champignon une association durable chez les Peltigera et les Nephromium, une association éphémère dans les céphalodies du Solorina saccata. Étudier la structure des Cyanophycées en symbiose durable dans les Peltigera et les Nephromium, celle des Cyanophycées emprisonnées dans les céphalodies du Solorina, la comparer à la structure des Cyanophycées libres, tel est le but de la présente Note.

Dangeard ${ }^{1}$, en 1894, a déjà signalé que les gonidies bleues des Lichens possédaient la structure des Cyanophycées libres

1. Dangeard (P.-A.), Recherches sur la structure des Lichens (C. R. Ac. Sc., 23 avril 1894). 


\section{$2 \mathrm{BHL}$ Biodiversity Heritage Library}

1916. "Séance Du 28 Janvier 1916." Bulletin de la Société botanique de France 63, 27-27. https://doi.org/10.1080/00378941.1916.10835945.

View This Item Online: https://www.biodiversitylibrary.org/item/8685

DOI: https://doi.org/10.1080/00378941.1916.10835945

Permalink: https://www.biodiversitylibrary.org/partpdf/159681

\section{Holding Institution}

Missouri Botanical Garden, Peter H. Raven Library

\section{Sponsored by}

Missouri Botanical Garden

\section{Copyright \& Reuse}

Copyright Status: Public domain. The BHL considers that this work is no longer under copyright protection.

This document was created from content at the Biodiversity Heritage Library, the world's largest open access digital library for biodiversity literature and archives. Visit BHL at https://www.biodiversitylibrary.org. 